\title{
Evidence for Multimagnon-Mediated Nuclear Spin Relaxation in the Intertwining Double-Chain Ferrimagnet $\mathrm{Ca}_{3} \mathrm{Cu}_{3}\left(\mathrm{PO}_{4}\right)_{4}$
}

\author{
Shoji Yamamoto, Hiromitsu Hori, Yuji Furukawa, Yusuke Nishisaka, \\ Yuzuru Sumida, Kiyotaka Yamada, and Ken-ichi Kumagai \\ Division of Physics, Hokkaido University, Sapporo 060-0810, Japan \\ Takayuki Asano \\ Department of Physics, Kyushu University, Fukuoka 812-8581, Japan \\ Yuji Inagaki \\ Department of Chemistry, Kyushu University, Fukuoka 812-8581, Japan
}

(Dated: June 25, 2018)

\begin{abstract}
The nuclear spin-lattice relaxation time $T_{1}$ of ${ }^{31} \mathrm{P}$ nuclei in the title compound is measured for the first time and interpreted in terms of a modified spin-wave theory. We establish a novel scenario for one-dimensional ferrimagnetic spin dynamics - it is three-magnon processes enhanced by exchange scattering, rather than Raman processes, that make the major contribution to $1 / T_{1}$.
\end{abstract}

PACS numbers: $76.50 .+\mathrm{g}, 76.60 .-\mathrm{k}, 75.50 . \mathrm{Gg}$

\section{INTRODUCTION}

Low-energy dynamics in one-dimensional (1D) quantum magnets is a long-standing problem and nuclear magnetic resonance (NMR) findings are often enlightening in this context. It was a fine collaboration that the NMR relaxation rates $1 / T_{1}$ and $1 / T_{2 \mathrm{G}}$ (Ref. 1) of the spin- $\frac{1}{2}$ antiferromagnet $\mathrm{Sr}_{2} \mathrm{CuO}_{3}$ illuminated multiplicative logarithmic corrections to the dynamic susceptibility ${ }^{2}$ in critical spin chains. $T_{1}$ measurements on the spin- $\frac{5}{2}$ antiferromagnet $\left(\mathrm{CH}_{3}\right)_{4} \mathrm{NMnCl}_{3}$ were a pioneering study in an attempt to detect the long-time diffusive spin dynamics in one dimension ${ }^{3}$ The diffusive contribution to $1 / T_{1}$ was observed in the spin- $\frac{1}{2}$ quantum limit $\$ .5$ and for spin-gapped antiferromagnets ${ }^{6}$ as well.

Antiferromagnets, whether critical or gapped, are thus vigorously studied, while little is known about ferrimagnetic, as well as ferromagnetic, dynamics. Recently numerous 1D ferrimagnets have been synthesized in an effort to design molecule-based ferromagnets. The static properties of various heterospin chains were correspondingly calculated, 7.8 .9 .10 .11 .12 .13 .14 but few effort has been devoted to exploring their dynamic features. Recent NMR observations of 1D ferrimagnets ${ }^{15.16 .17}$ have thus been motivated and have indeed stimulated the theoretical interest in them. The most pioneering $T_{1}$ measurements, $\stackrel{15}{\stackrel{15}{5}}$ performed on the metal-radical hybrid compound $\mathrm{Mn}\left(\mathrm{C}_{5} \mathrm{H}_{2} \mathrm{O}_{2} \mathrm{~F}_{6}\right)_{2} \mathrm{C}_{10} \mathrm{H}_{17} \mathrm{~N}_{2} \mathrm{O}_{2}$, were interpreted in terms of solitonic excitations at low temperatures and based on the spin-diffusion model at high temperatures. However, this material has rather large exchange interactions and its magnetic susceptibility $(\chi)$ times temperature $(T)$ exhibits no ferrimagnetic minimum at a measurable temperature ${ }^{18}$ The nonnegligible single-ion anisotropy and the spread magnetic moment over the radical may also blur the intrinsic ferrimagnetic features. The family of manganeseporphyrin-

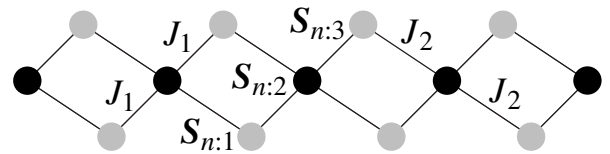

FIG. 1: $\mathrm{Cu}^{\mathrm{II}}$ trimeric chains in $\mathrm{Ca}_{3} \mathrm{Cu}_{3}\left(\mathrm{PO}_{4}\right)_{4}$. The strongly coupled $\mathrm{Cu}^{\mathrm{II}}$ trimer consists of a central square planar $\mathrm{Cu}(1)$ ion (black circle) and two pyramidal $\mathrm{Cu}(2)$ ions (gray circles).
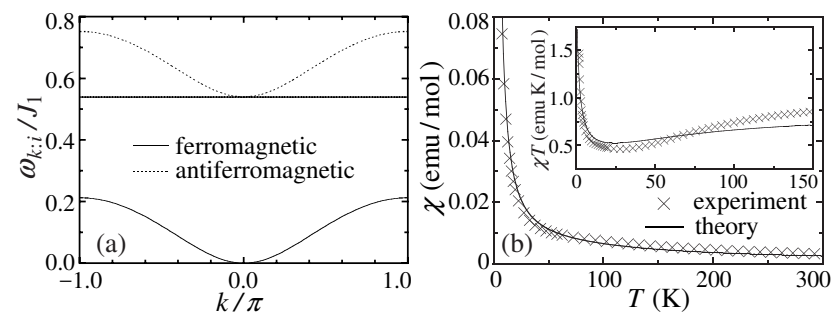

FIG. 2: (a) Dispersion relations of the spin-wave excitations. (b) Modified spin-wave calculations of the susceptibility compared with experimental findings at $H=0.1 \mathrm{~T}$.

based ferrimagnets, whose exchange interactions are somewhat smaller, was also observed through NMR, 17 but the complicated crystalline structure restricted the analysis to solving the magnetic configuration. ${ }^{1} \mathrm{H}$ NMR findings 16 on the bimetallic chain compound $\mathrm{NiCu}\left(\mathrm{C}_{7} \mathrm{H}_{6} \mathrm{~N}_{2} \mathrm{O}_{6}\right)\left(\mathrm{H}_{2} \mathrm{O}\right)_{3} \cdot 2 \mathrm{H}_{2} \mathrm{O}$ implied a spin-wave relaxation scenario, but the averaging effect over the numerous protons masks the low-temperature dynamics.

In such circumstances, we perform an NMR study on the homometallic chain compound $\mathrm{Ca}_{3} \mathrm{Cu}_{3}\left(\mathrm{PO}_{4}\right)_{4}$ (Fig. 11), which is a novel ferrimagnet of topological origin 19 This collaboration aims at verifying a newly developed modified spin-wave nuclear magnetic relaxation theory $\stackrel{20}{2}$ Two of the present authors have recently demonstrated that $1 / T_{1}$ in $1 \mathrm{D}$ heterometallic ferrimagnets may significantly be enhanced by exchange interactions, in an 
attempt to interpret the novel NMR observations ${ }^{16}$ for $\mathrm{NiCu}\left(\mathrm{C}_{7} \mathrm{H}_{6} \mathrm{~N}_{2} \mathrm{O}_{6}\right)\left(\mathrm{H}_{2} \mathrm{O}\right)_{3} \cdot 2 \mathrm{H}_{2} \mathrm{O}$, which are hardly understandable within the usual Raman relaxation scheme. However, all the findings ${ }^{21}$ were far from conclusive to a possibility of more than two magnons mediating the proton spin relaxation. Since only the ${ }^{1} \mathrm{H}$ nuclei were practically available as probes in $\mathrm{NiCu}\left(\mathrm{C}_{7} \mathrm{H}_{6} \mathrm{~N}_{2} \mathrm{O}_{6}\right)\left(\mathrm{H}_{2} \mathrm{O}\right)_{3} \cdot 2 \mathrm{H}_{2} \mathrm{O}$, the crystal water of nuisance and the resultant ill-behaving spin-echo recovery curve with increasing field at low temperatures restricted the $T_{1}$ analysis to rather high temperatures $T \gtrsim 60 \mathrm{~K}$. $\mathrm{Ca}_{3} \mathrm{Cu}_{3}\left(\mathrm{PO}_{4}\right)_{4}$ contains $\mathrm{P}$ atoms as efficient probes. The well-isolated $\mathrm{Cu}^{\mathrm{II}}$ chains with $\mathrm{Ca}$ columns in between, no single-ion anisotropy of the $\mathrm{Cu}^{\mathrm{II}}$ spins, and the ferrimagnetic minimum of $\chi T$ at a moderate temperature [see Fig. 2(b)] furthermore guarantee this material to be a fine stage of 1D ferrimagnetic dynamics.

\section{MULTIMAGNON-MEDIATED NUCLEAR SPIN RELAXATION}

We describe $\mathrm{Ca}_{3} \mathrm{Cu}_{3}\left(\mathrm{PO}_{4}\right)_{4}$ by the Hamiltonian,

$$
\begin{aligned}
\mathcal{H}= & \sum_{n=1}^{N}\left[J_{1}\left(\boldsymbol{S}_{n: 1} \cdot \boldsymbol{S}_{n: 2}+\boldsymbol{S}_{n: 2} \cdot \boldsymbol{S}_{n: 3}\right)\right. \\
& +J_{2}\left(\boldsymbol{S}_{n+1: 1} \cdot \boldsymbol{S}_{n: 2}+\boldsymbol{S}_{n: 2} \cdot \boldsymbol{S}_{n-1: 3}\right) \\
& \left.-g \mu_{\mathrm{B}} H\left(S_{n: 1}^{z}+S_{n: 2}^{z}+S_{n:}^{z}\right)\right]
\end{aligned}
$$

where each $\mathrm{Cu}(1)$ ion is antiferromagnetically coupled to four $\mathrm{Cu}(2)$ ions in an applied field $(H)$ (see Fig. 1). We set $J_{1} / k_{\mathrm{B}}$ and $J_{2} / k_{\mathrm{B}}$ equal to $100 \mathrm{~K}$ and $8 \mathrm{~K}$, respectively 19 Employing the Holstein-Primakoff transformation, ${ }^{22}$ we expand the Hamiltonian with respect to $1 / S$ as

$$
\mathcal{H}=-2 S^{2}\left(J_{1}+J_{2}\right) N+\mathcal{H}_{1}+\mathcal{H}_{0}+O\left(S^{-1}\right)
$$

where $\mathcal{H}_{i}$ contains the $O\left(S^{i}\right)$ terms. $\mathcal{H}_{1}$ describes linear spin-wave excitations and is diagonalized in the momentum space as

$$
\mathcal{H}_{1}=-\frac{3}{2}\left(J_{1}+J_{2}\right)+\sum_{k}\left(\omega_{k}+\sum_{i=1}^{3} \omega_{k: i} \alpha_{k: i}^{\dagger} \alpha_{k: i}\right)
$$

where $\alpha_{k: i}^{\dagger}$ creates a spin wave of ferromagnetic $(i=1,2)$ or antiferromagnetic $(i=3)$ aspect, whose excitation energy is given by

$$
\begin{aligned}
& \omega_{k: 1}=\omega_{k}-\frac{S}{2}\left(J_{1}+J_{2}\right)+g \mu_{\mathrm{B}} H, \\
& \omega_{k: 2}=\left(J_{1}+J_{2}\right) S+g \mu_{\mathrm{B}} H, \\
& \omega_{k: 3}=\omega_{k}+\frac{S}{2}\left(J_{1}+J_{2}\right)-g \mu_{\mathrm{B}} H,
\end{aligned}
$$

with

$$
\omega_{k}=\frac{S}{2} \sqrt{\left(J_{1}+J_{2}\right)^{2}+32 J_{1} J_{2} \sin ^{2} \frac{k}{2}}
$$

[see Fig. 2(a)]. $\quad \mathcal{H}_{0}$ gives two-body interactions and makes a crucial contribution to nuclear spin-lattice relaxation. The $O\left(S^{-1}\right)$ terms are neglected in the following. The dispersive branches $\omega_{k: 1}$ and $\omega_{k: 3}$ are reminiscent of the dual excitations in alternating-spin chains, ${ }^{23}$ whereas the flat band $\omega_{k: 2}$, describing intratrimer excitations, is peculiar to the present system.

Our way ${ }^{12.24}$ of modifying the conventional spin-wave theory is distinct from the original idea proposed by Takahashi $^{25}$ and Hirsch et al. ${ }^{26}$ Their way of suppressing the divergent sublattice magnetizations consists of diagonalizing the Hamiltonian together with a Lagrange multiplier subject to zero staggered magnetization. The thus-obtained energy spectrum depends on temperature and fails, for instance, to reproduce the Schottky-peaked specific heat. Seeking after better thermodynamics, we diagonalize the bare Hamiltonian and then minimize the free energy with a Lagrange multiplier subject to zero staggered magnetization. ${ }^{27}$ The thus-calculated $\chi$ is in good agreement with observations [Fig. 2(b)].

The hyperfine interaction between a ${ }^{31} \mathrm{P}$ nucleus and $\mathrm{Cu}^{\mathrm{II}}$ spins consists of isotropic Fermi contact and anisotropic dipolar coupling and is defined as

$$
\mathcal{H}_{\mathrm{hf}}=g \mu_{\mathrm{B}} \hbar \gamma_{\mathrm{N}} I^{+} \sum_{n} \sum_{i=1}^{3}\left(\frac{1}{2} A_{n: i}^{-} S_{n: i}^{-}+A_{n: i}^{z} S_{n: i}^{z}\right) .
$$

$\mathcal{H}_{0}$ and $\mathcal{H}_{\mathrm{hf}}$ are both much smaller than $\mathcal{H}_{1}$ and may be regarded as perturbations to the linear spin-wave system. When we calculate up to second order in $\mathcal{V} \equiv \mathcal{H}_{0}+\mathcal{H}_{\mathrm{hf}}$, the probability of a nuclear spin being scattered from the state of $I^{z}=m$ to that of $I^{z}=m+1$ is given by

$$
W=\frac{2 \pi}{\hbar} \sum_{f}\left|\left\langle f\left|\mathcal{V}+\sum_{m(\neq i)} \frac{\mathcal{V}|m\rangle\langle m| \mathcal{V}}{E_{i}-E_{m}}\right| i\right\rangle\right|^{2} \delta\left(E_{i}-E_{f}\right)
$$

where $i$ and $f$ denote the initial and final states of the unperturbed electronic-nuclear spin system. Then we obtain $T_{1}=(I-m)(I+m+1) / 2 W$. Considering the significant difference between the nuclear and electronic energy scales at moderate fields and assuming the Fourier components of the coupling tensors to have little momentum dependence as $\sum_{n} e^{\mathrm{i} k(n+i / 2-1)} A_{n: i}^{\lambda} \equiv A_{k, i}^{\lambda} \simeq A_{i}^{\lambda}$, the Raman and three-magnon relaxation rates read

$$
\begin{aligned}
\frac{1}{T_{1}^{(2)}} & \simeq \frac{2\left(g \mu_{\mathrm{B}} \hbar \gamma_{\mathrm{N}}\right)^{2}}{\hbar N} \sum_{k_{1}} \sum_{\sigma= \pm} \sum_{i=1,3}\left|W_{i i}\left(k_{1}, \sigma k_{2}^{(i)}\right)\right|^{2} \\
& \times\left(\bar{n}_{k_{1}: i}+1\right) \bar{n}_{k_{2}^{(i)}: i}\left|\frac{\mathrm{d} \omega_{k: i}}{\mathrm{~d} k}\right|_{k=k_{2}^{(i)}}^{-1}, \\
\frac{1}{T_{1}^{(3)}} & \simeq \frac{\left(g \mu_{\mathrm{B}} \hbar \gamma_{\mathrm{N}}\right)^{2}}{16 \hbar S N^{2}} \sum_{k_{1}, k_{2}} \sum_{\sigma= \pm}\left[2\left|W_{111}\left(k_{1}, k_{2}, \sigma k_{3}^{(1)}\right)\right|^{2}\right. \\
& \times \bar{n}_{k_{1}: 1}\left(\bar{n}_{k_{2}: 1}+1\right)\left(\bar{n}_{k_{3}^{(1)}: 1}+1\right)\left|\frac{\mathrm{d} \omega_{k: 1}}{\mathrm{~d} k}\right|_{k=k_{3}^{(1)}}^{-1} \\
& +\left|W_{132}\left(k_{1}, k_{2}, \sigma k_{3}^{(2)}\right)\right|^{2} \bar{n}_{k_{1}: 1} \bar{n}_{k_{2}: 3}\left(\bar{n}_{k_{3}^{(2)}: 2}+1\right)
\end{aligned}
$$




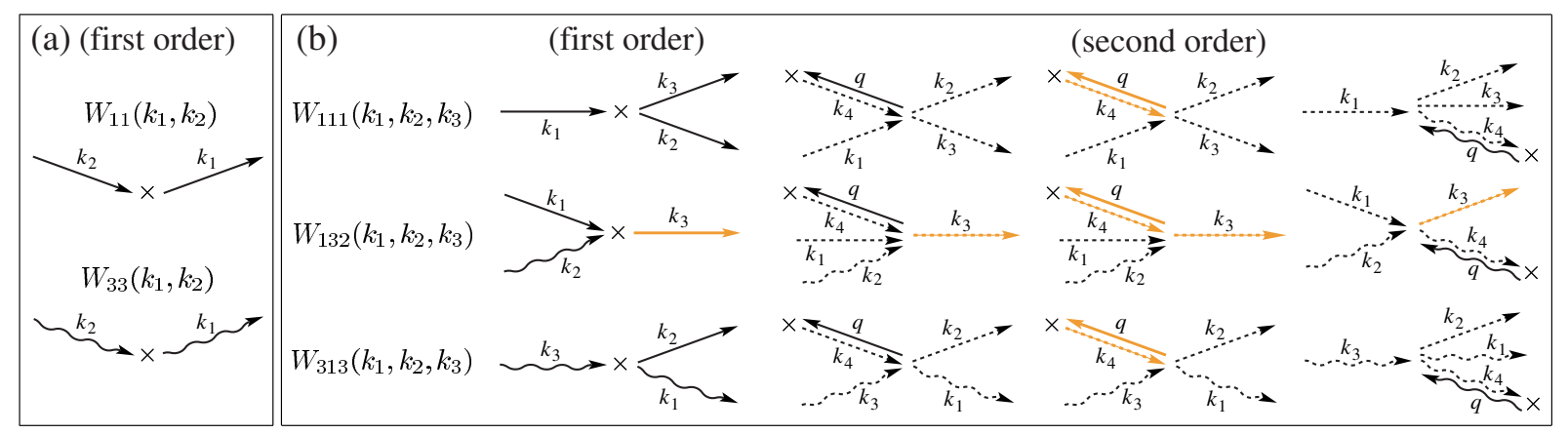

FIG. 3: (Color online) Various nuclear spin-lattice relaxation processes. Spin waves which are emitted in the first-order mechanism (solid arrows) flip a nuclear spin $(x)$ via the hyperfine interaction, whereas four-magnon exchange correlations (dotted arrows) thermally scatter a first-order virtual spin wave with $q=-k_{4}$, where ferromagnetic spin waves are drawn by black $\left(\omega_{k: 1}\right)$ and colored $\left(\omega_{k: 2}\right)$ straight arrows, while antiferromagnetic ones $\left(\omega_{k: 3}\right)$ by wavy arrows. (a) First-order Raman processes. (b) First- and second-order three-magnon processes, which are inseparable in nonlinear equations.

$$
\begin{aligned}
& \times\left|\frac{\mathrm{d} \omega_{k: 2}}{\mathrm{~d} k}\right|_{k=k_{3}^{(2)}}^{-1}+\left|W_{313}\left(k_{1}, k_{2}, \sigma k_{3}^{(3)}\right)\right|^{2} \\
& \left.\times\left(\bar{n}_{k_{1}: 3}+1\right)\left(\bar{n}_{k_{2}: 1}+1\right) \bar{n}_{k_{3}^{(3)}: 3}\left|\frac{\mathrm{d} \omega_{k: 3}}{\mathrm{~d} k}\right|_{k=k_{3}^{(3)}}^{-1}\right]
\end{aligned}
$$

where $\bar{n}_{k: i} \equiv\left\langle\alpha_{k: i}^{\dagger} \alpha_{k: i}\right\rangle$ is the thermal distribution function of modified spin waves, while $k_{2}^{(i)}$ and $k_{3}^{(i)}$ are determined through $\omega_{k_{1}: i}-\omega_{k_{2}^{(i)}: i}-\hbar \omega_{\mathrm{N}}=0, \omega_{k_{3}^{(1)}: 1}+\omega_{k_{2}: 1}-$ $\omega_{k_{1}: 1}-\hbar \omega_{\mathrm{N}}=0, \omega_{k_{3}^{(2)}: 2}-\omega_{k_{2}: 3}-\omega_{k_{1}: 1}-\hbar \omega_{\mathrm{N}}=0$, and $\omega_{k_{3}^{(3)}: 3}-\omega_{k_{2}: 1}-\omega_{k_{1}: 3}+\hbar \omega_{\mathrm{N}}=0 . W_{i i}\left(k_{1}, k_{2}\right)$ and $W_{i j l}\left(k_{1}, k_{2}, k_{3}\right)$ are diagrammatically represented in Fig. 3] Within the first-order mechanism containing a nuclear spin in direct contact with spin waves via the hyperfine interaction, any multi-magnon relaxation rate is much smaller than the Raman one. However, some of multimagnon processes make a significant contribution to $1 / T_{1}$ through the second-order mechanism, where a nuclear spin flips with the help of virtual spin waves which are then scattered thermally via the four-magnon exchange interaction. We consider the leading second-order relaxation, that is, exchange-scattering-induced three-magnon processes, as well as the first-order relaxation. Secondorder single-magnon and Raman relaxation processes, containing three and two virtual magnons, respectively, are much more accidental due to the momentum conservation and much less contributive due to the magnon series damping. As for four-magnon processes, the firstorder relaxation is nonexistent to begin with, whereas the second-order one originates in the six-magnon exchange interaction and contains two virtual magnons. Thus and thus, all other higher-order processes have no significant effect on the relaxation scenario.

\section{EXPERIMENTAL TEST}

Now we present ${ }^{31} \mathrm{P}$ NMR spectra for powder samples of $\mathrm{Ca}_{3} \mathrm{Cu}_{3}\left(\mathrm{PO}_{4}\right)_{4}$ in Fig. [4 The crystallographically

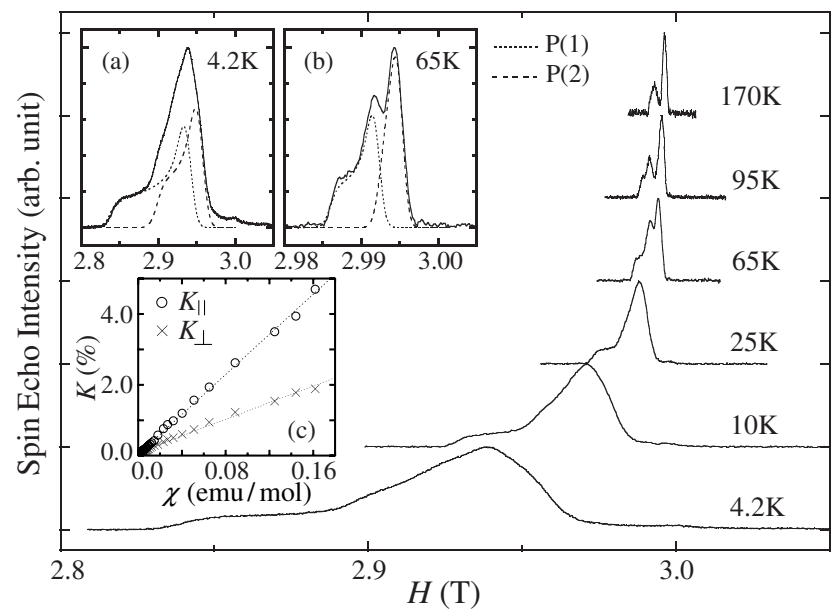

FIG. 4: ${ }^{31} \mathrm{P}$ NMR spectra measured at $51.711 \mathrm{MHz}$. They can be decomposed into typical powder patterns [the insets (a) and (b)], originating in the $\mathrm{P}(1)$ (dotted lines) and $\mathrm{P}(2)$ (broken lines) sites, and the NMR shift for the latter is plotted as a function of the susceptibility at $H=3 \mathrm{~T}$ [the inset (c)].

inequivalent $\mathrm{P}$ sites, labeled $\mathrm{P}(1)$ and $\mathrm{P}(2)$, give two distinct lines, each of which has a characteristic shape of the anisotropic powder pattern, as is revealed in the insets (a) and (b). Both lines broaden and shift to lower field with decreasing temperature, implying that contact as well as dipolar terms exist in the hyperfine field on the $\mathrm{P}$ nuclei. The NMR shifts are thus anisotropic and their parallel $\left(K_{\|}\right)$and perpendicular $\left(K_{\perp}\right)$ components for the $\mathrm{P}(2)$ sites are plotted as functions of the susceptibility at $H=3 \mathrm{~T}$ in the inset (c). If we roughly estimate the hyperfine coupling between each $\mathrm{P}(2)$ nucleus and the nearest-neighbor $\mathrm{Cu}^{\mathrm{II}}$ ion through the relations $A_{\perp}=$ $N_{\mathrm{A}} \mu_{\mathrm{B}} \mathrm{d} K_{\perp} / \mathrm{d} \chi$ and $A_{\|}=N_{\mathrm{A}} \mu_{\mathrm{B}} \mathrm{d} K_{\|} / \mathrm{d} \chi$, where $N_{\mathrm{A}}$ is the Avogadro number, we find that $A_{\perp}=0.66 \mathrm{kOe} / \mu_{\mathrm{B}}$ and $A_{\|}=1.58 \mathrm{kOe} / \mu_{\mathrm{B}}$. In order to reveal the low-frequency spin dynamics, we measure $T_{1}$ for the $\mathrm{P}(2)$ sites through the saturation-recovery method.

Temperature dependences of $1 / T_{1}$ are shown in Fig. [5 In each $\mathrm{Cu}^{\mathrm{II}}$ trimer unit, the two spins $\boldsymbol{S}_{n: 1}$ and 


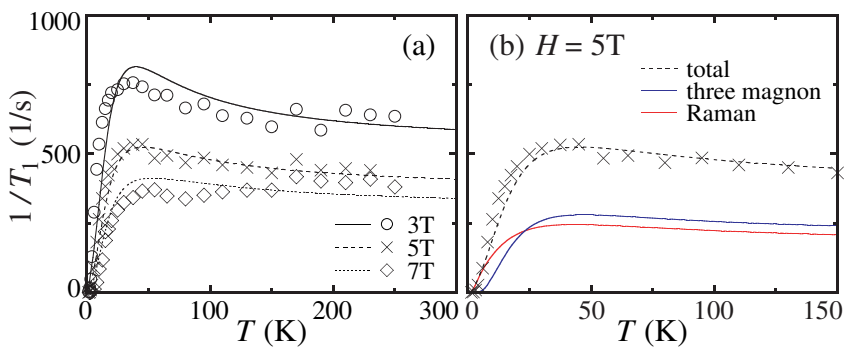

FIG. 5: (Color online) Experimental (symbols) and theoretical (lines) findings of $1 / T_{1}$ as functions of temperature. $1 / T_{1}^{(2)}$ and $1 / T_{1}^{(3)}$ are also plotted in (b).
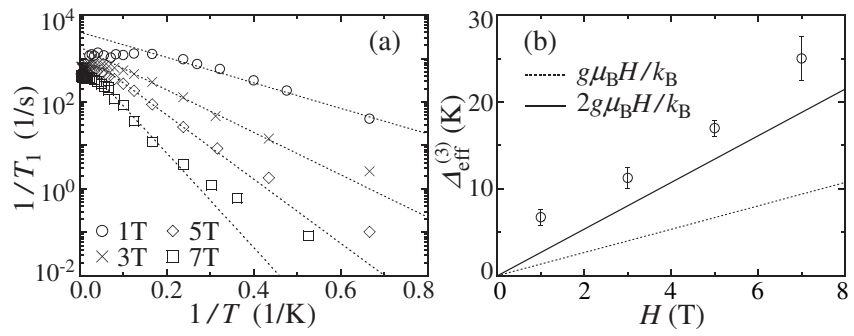

FIG. 6: (a) Semilog plots of $1 / T_{1}$ as functions of $1 / T$. Dotted lines are guides to the probable exponential behavior $1 / T_{1} \propto \mathrm{e}^{-\Delta_{\text {eff }}^{(3)} / T}$ at each field. (b) The thus-extracted $\Delta_{\text {eff }}^{(3)}$ as a function of field. The two slopes, $\Delta_{\text {eff }}^{(3)}=g \mu_{\mathrm{B}} H / k_{\mathrm{B}}$ and $\Delta_{\text {eff }}^{(3)}=2 g \mu_{\mathrm{B}} H / k_{\mathrm{B}}$, are shown for reference.

$\boldsymbol{S}_{n: 2}$ are almost equidistant from the nearby $\mathrm{P}(2)$ atom, whereas the other one $\boldsymbol{S}_{n: 3}$ is much more distant from that 28 Therefore, the isotropic coupling constants are taken as $A_{1}^{-}=A_{2}^{-} \equiv A^{-}$and $A_{3}^{-}=0$. Since dipolar interactions are also sensitive to the location of correlating moments, the anisotropic coupling constants may be taken similarly as $A_{1}^{z}=A_{2}^{z} \equiv A^{z}$ and $A_{3}^{z}=0$. Then we set $A^{-}$and $A^{z}$ equal to $0.85 \mathrm{kOe} / \mu_{\mathrm{B}}$ and $1.20 \mathrm{kOe} / \mu_{\mathrm{B}}$, respectively, which are both consistent well with the experimental findings $A_{\perp}=0.66 \mathrm{kOe} / \mu_{\mathrm{B}}$ and $A_{\|}=1.58 \mathrm{kOe} / \mu_{\mathrm{B}}$. Considering that recent electronspin-resonance measurements of this compound 29 have yield temperature-dependent and anisotropic $g$ values ( $g_{\|}>g_{\perp} \simeq 2$ ), the $A_{\|} / A_{\perp}$ value may be closer to the theoretical parametrization. We are further convinced of the coupling constants employed finding the nearest $\mathrm{Cu}(1)$ $\mathrm{P}(2)$ distance to be about $\left(1 / A^{z}\right)^{1 / 3}=2.5 \AA$, which is in excellent agreement with the crystalline structure 28 The thus-calculated $1 / T_{1}=1 / T_{1}^{(2)}+1 / T_{1}^{(3)}$ reproduces the observations pretty well. The exchange-scatteringenhanced three-magnon relaxation rate generally grows into a major contribution to $1 / T_{1}$ with increasing temperature and decreasing field. While both $1 / T_{1}^{(2)}$ and $1 / T_{1}^{(3)}$ exhibit an exponential behavior at low temperatures, their activation energies, referred to as $k_{\mathrm{B}} \Delta_{\text {eff }}^{(2)}$ and $k_{\mathrm{B}} \Delta_{\text {eff }}^{(3)}$, respectively, look different. At moderately low

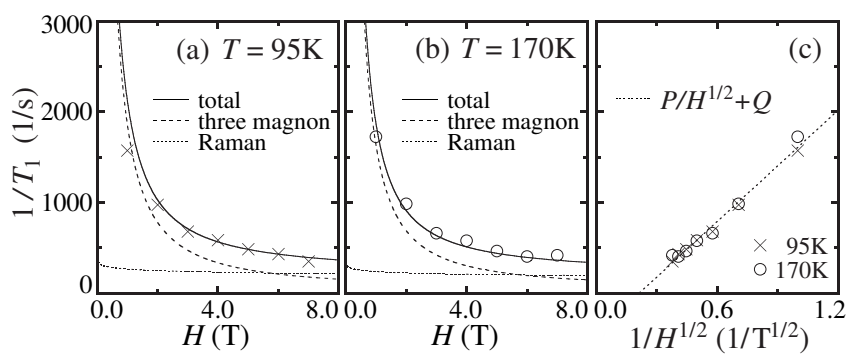

FIG. 7: Experimental (symbols) and theoretical (lines) findings of $1 / T_{1}$ as functions of field at $95 \mathrm{~K} \mathrm{(a)}$ and $170 \mathrm{~K}(\mathrm{~b})$, where $1 / T_{1}^{(2)}$ and $1 / T_{1}^{(3)}$ are also plotted. The observations are replotted as functions of $1 / \sqrt{H}$ and fitted to an expression $1 / T_{1}=P / \sqrt{H}+Q(\mathrm{c})$.

temperatures and weak fields, $\hbar \omega_{\mathrm{N}} \ll k_{\mathrm{B}} T \ll J_{2}$, Eq. (8) reads

$\frac{1}{T_{1}^{(2)}} \simeq \frac{J_{1}+J_{2}}{2 \pi \hbar S J_{1} J_{2}}\left(g \mu_{\mathrm{B}} \hbar \gamma_{\mathrm{N}} A^{z}\right)^{2} \mathrm{e}^{-g \mu_{\mathrm{B}} H / k_{\mathrm{B}} T} K_{0}\left(\frac{\hbar \omega_{\mathrm{N}}}{2 k_{\mathrm{B}} T}\right)$,

where $K_{0}$ is the modified Bessel function of the second kind and behaves as $K_{0}\left(\hbar \omega_{\mathrm{N}} / 2 k_{\mathrm{B}} T\right) \simeq 0.80908-$ $\ln \left(\hbar \omega_{\mathrm{N}} / k_{\mathrm{B}} T\right)$. Thus we learn that $\Delta_{\mathrm{eff}}^{(2)} \simeq g \mu_{\mathrm{B}} H / k_{\mathrm{B}}$. Equation (9) is much less analyzable, but Fig. [5(b) claims that $\Delta_{\text {eff }}^{(2)}<\Delta_{\text {eff }}^{(3)}$. Figure 6 brings the next leading exponential behavior $1 / T_{1} \propto \mathrm{e}^{-\Delta_{\text {eff }}^{(3)} / T}$ to light, because Eq. (10) is valid for $T \ll J_{2} / k_{\mathrm{B}}=8 \mathrm{~K}$. $\Delta_{\text {eff }}^{(3)}$ looks like $2 g \mu_{\mathrm{B}} H / k_{\mathrm{B}}$ or more, rather than $g \mu_{\mathrm{B}} H / k_{\mathrm{B}}$. The twomagnon-mediated nuclear spin relaxation is mainly given by $W_{11}\left(k_{1}, k_{2}\right)$, where a ferromagnetic spin wave of energy $\omega_{k_{1}: 1}$ is created and that of energy $\omega_{k_{2}: 1} \simeq \omega_{k_{1}: 1}$ is destructed, while the three-magnon-mediated one by $W_{111}\left(k_{1}, k_{2}, k_{3}\right)$, where two net spin waves of energy $\omega_{k_{2}: 1}$ and $\omega_{k_{3}: 1}$ are created and that of energy $\omega_{k_{1}: 1} \simeq$ $\omega_{k_{2}: 1}+\omega_{k_{3}: 1}$ is destructed. Therefore, Raman processes are activated by the energy $\omega_{k=0: 1}=g \mu_{\mathrm{B}} H$, which is consistent with Eq. (10), whereas three-magnon ones roughly by the energy $2 \omega_{k=0: 1}=2 g \mu_{\mathrm{B}} H$, which may look somewhat larger due to the complicated field dependence lying in the second-order mechanism.

Field dependences of $1 / T_{1}$ at higher temperatures more impress on us the significance of three-magnon processes. Figures 7(a) and 7(b) show that the accelerated relaxation with decreasing field can never be explained by the Raman scheme but should be attributed to exchange-scattering-enhanced three-magnon processes. The spin-diffusion model may be mentioned in this context. Diffusion-dominated 1D spin dynamics gives $1 / T_{1}$ of the form $P / \sqrt{H}+Q, 30$ where the first and second terms come from transverse and longitudinal spin fluctuations, respectively, and are both positive. Figure 7(c) shows that the present observations fitted to the diffusive law result in negative $Q$. We do not exclude a possibility of diffusive dynamics appearing 
in 1D ferrimagnets as well, but a distinct field dependence of the second-order relaxation mechanism masks such a moderate field effect in the present case. It was in $\mathrm{AgVP}_{2} \mathrm{~S}_{6}$ rather than in the most familiar Haldanegap antiferromagnet $\mathrm{Ni}\left(\mathrm{C}_{2} \mathrm{H}_{8} \mathrm{~N}_{2}\right)_{2} \mathrm{NO}_{2} \mathrm{ClO}_{4}$, whose excitation spectrum drastically varies with increasing field, that integral-spin diffusive correlations were observed ${ }^{6}$ Exchange-scattering-induced three-magnon processes are sensitive to an excitation gap and their contribution to $1 / T_{1}$ is strongly suppressed, for example, by slight magnetic anisotropy. There are indeed some indications of spin diffusion ${ }^{15}$ in the ferrimagnetic chain compound $\mathrm{Mn}\left(\mathrm{C}_{5} \mathrm{H}_{2} \mathrm{O}_{2} \mathrm{~F}_{6}\right){ }_{2} \mathrm{C}_{10} \mathrm{H}_{17} \mathrm{~N}_{2} \mathrm{O}_{2}$ with nonnegligible singleion anisotropy. 18

\section{CONCLUDING REMARKS}

We have performed NMR measurements on the topological ferrimagnet $\mathrm{Ca}_{3} \mathrm{Cu}_{3}\left(\mathrm{PO}_{4}\right)_{4}$ and have confirmed a novel scenario for $1 \mathrm{D}$ spin dynamics-multimagnonmediated nuclear spin relaxation, by showing the parametrization to be crystallographically convincing, revealing that the relaxation is activated by twice the gap rather than the gap itself at low temperatures, while it is remarkably accelerated with decreasing field, and pointing out the irrelevance of the spin-diffusion model. Indeed pioneering $T_{1}$ observations on the layered ferromag- net $\mathrm{CrCl}_{3}$ (Ref. 31) and the coupled-chain antiferromagnet $\mathrm{CsMnCl}_{3} \cdot 2 \mathrm{H}_{2} \mathrm{O}$ (Ref. 32) claimed to have detected three-magnon processes, but they were both, in some sense, classical findings under the 3D long-range order 33 Our findings are literally 1D quantum spin relaxation beyond the Raman mechanism, which were obtained through an elaborately modified spin-wave theory. ${ }^{27}$

The bond-alternating homometallic chain compound $\mathrm{Cu}\left(\mathrm{C}_{5} \mathrm{ClH}_{4} \mathrm{~N}\right)_{2}\left(\mathrm{~N}_{3}\right)_{2}$ (Ref. 34) is another anisotropy-free ferrimagnet of topological origin 35 and therefore NMR measurements on it are highly encouraged. Since 1D ferromagnets, which can be regarded as low-energy sectors of 1D ferrimagnets, may also play this fascinating scenario, more understanding will come with further experiments, for instance, on the spin- $\frac{1}{2}$ ferromagnetic chain compound $\left(\mathrm{CH}_{3}\right)_{4} \mathrm{NCuCl}_{3}$ 年 The quasi-one-dimensional mixed-spin ferromagnet $\mathrm{MnNi}\left(\mathrm{NO}_{2}\right)_{4}\left(\mathrm{C}_{2} \mathrm{H}_{8} \mathrm{~N}_{2}\right)_{2}$ (Ref. 37) is also highly interesting in this context, whose lowenergy spectrum consists of two dispersive ferromagnetic excitation branches. ${ }^{38.39}$

\section{Acknowledgments}

This work was supported by the Ministry of Education, Culture, Sports, Science, and Technology of Japan.
1 M. Takigawa, O. A. Starykh, A. W. Sandvik and R. R. P. Singh: Phys. Rev. B 56 (1997) 13681.

2 O. A. Starykh, R. R. P. Singh and A. W. Sandvik: Phys. Rev. Lett. 78 (1997) 539.

${ }^{3}$ F. Borsa and M. Mali: Phys. Rev. B 9 (1974) 2215.

4 M. Takigawa, N. Motoyama, H. Eisaki and S. Uchida: Phys. Rev. Lett. 76 (1996) 4612.

5 J. Kikuchi, N. Kurata, K. Motoya, T. Yamauchi and Y. Ueda: J. Phys. Soc. Jpn. 70 (2001) 2765.

6 M. Takigawa, T. Asano, Y. Ajiro, M. Mekata and Y. J. Uemura: Phys. Rev. Lett. 76 (1996) 2173.

7 M. Drillon, E. Coronado, R. Georges, J. C. Gianduzzo and J. Curely: Phys. Rev. B 40 (1989) 10992.

8 A. K. Kolezhuk, H.-J. Mikeska and S. Yamamoto: Phys. Rev. B 55 (1997) R3336.

9 G.-S. Tian: Phys. Rev. B 56 (1997) 5355.

10 S. Brehmer, H.-J. Mikeska and S. Yamamoto: J. Phys.: Condens. Matter 9 (1997) 3921.

11 S. K. Pati, S. Ramasesha and D. Sen: Phys. Rev. B 55 (1997) 8894; J. Phys.: Condens. Matter 9 (1997) 8707.

12 S. Yamamoto and T. Fukui: Phys. Rev. B 57 (1998) R14008.

13 N. B. Ivanov, Phys. Rev. B 57, R14024 (1998); ibid. 62, 3271 (2000).

14 S. Yamamoto, Phys. Rev. B 59, 1024 (1999).

15 F. Ferraro, D. Gatteschi, R. Sessoli and M. Corti: J. Am. Chem. Soc. 113 (1991) 8410; F. Ferraro, D. Gatteschi, A. Rettori and M. Corti: Mol. Phys. 85 (1995) 1073.

16 N. Fujiwara and M. Hagiwara: Solid State Commun. 113
(2000) 433.

17 M. Fardis, G. Diamantopoulos, G. Papavassiliou, K. Pokhodnya, J. S. Miller, D. K. Rittenberg and C. Christides: Phys. Rev. B 66 (2002) 064422.

18 A. Caneschi, D. Gatteschi, P. Rey and R. Sessoli: Inorg. Chem. 27 (1988) 1756.

19 M. Drillon, M. Belaiche, P. Legoll, J. Aride, A. Boukhari and A. Moqine: J. Magn. Magn. Mat. 128 (1993) 83.

20 H. Hori and S. Yamamoto: J. Phys. Soc. Jpn. 73 (2004) 1453.

21 H. Hori and S. Yamamoto: J. Phys.: Condens. Matter 16 (2004) 9023.

22 T. Holstein and H. Primakoff: Phys. Rev. 58 (1940) 1098.

23 S. Yamamoto, S. Brehmer and H.-J. Mikeska: Phys. Rev. B 57 (1998) 13610.

24 S. Yamamoto, T. Fukui, K. Maisinger and U. Schollwöck, J. Phys.: Condens. Matter 10 (1998) 11033.

25 M. Takahashi: Phys. Rev. B 40 (1989) 2494.

26 J. E. Hirsch and S. Tang: Phys. Rev. B 40 (1989) 4769; S. Tang, M. E. Lazzouni and J. E. Hirsch: ibid. 40 (1989) 5000 .

27 S. Yamamoto: Phys. Rev. B 69 (2004) 064426.

28 J. B. Anderson, E. Kostiner and F. A. Ruszala: J. Solid State Chem. 39 (1981) 29.

29 S. Okubo, H. Ishikawa, S. Kimura, H. Ohta, Y. Inagaki, A. A. Belik, M. Azuma and M. Takano: to be published in AIP Conf. Proc..

30 D. Hone, C. Scherer and F. Borsa: Phys. Rev. B 9 (1974) 965. 
31 A. Narath and A. T. Fromhold, Jr.: Phys. Rev. Lett. 17 (1966) 354.

32 H. Nishihara, W. J. M. de Jonge and T. de Neef: Phys. Rev. B 12 (1975) 5325.

33 P. Pincus: Phys. Rev. Lett. 16 (1966) 398; D. Beeman and P. Pincus: Phys. Rev. 166 (1968) 359.

34 A. Escuer, R. Vicente, M. S. El Fallah, M. A. S. Goher and F. A. Mautner: Inorg. Chem. 37 (1998) 4466.

35 T. Nakanishi and S. Yamamoto: Phys. Rev. B 65 (2002)
214418.

36 C. P. Landee and R. D. Willett: Phys. Rev. Lett. 43 (1979) 463.

37 O. Kahn, E. Bakalbassis, C. Mathonière, M. Hagiwara, K. Katsumata and L. Ouahab, Inorg. Chem. 36 (1997) 1530.

${ }^{38}$ N. Fukushima, A. Honecker, S. Wessel and W.Brenig: Phys. Rev. B 69 (2004) 174430.

39 S. Yamamoto and H. Hori: Phys. Rev. B 72 (2005) 054423. 